\title{
B lymphocytes protect islet $\beta$ cells in diabetes-prone NOD mice treated with imatinib
}

\author{
Christopher S. Wilson, ${ }^{1}$ Jason M. Spaeth, ${ }^{2}$ Jay Karp, ${ }^{2}$ Blair T. Stocks, ${ }^{3}$ Emilee M. Hoopes, ${ }^{1}$ \\ Roland W. Stein, ${ }^{2}$ and Daniel J. Moore ${ }^{1,3}$ \\ 'Department of Pediatrics, Ian Burr Division of Endocrinology and Diabetes, Vanderbilt University Medical Center, \\ Nashville, Tennessee, USA. 'Department of Molecular Physiology and Biophysics, Vanderbilt University, Nashville, \\ Tennessee, USA. 'Department of Pathology, Microbiology and Immunology, Vanderbilt University Medical Center, \\ Nashville, Tennessee, USA.
}

Imatinib (Gleevec) reverses type 1 diabetes (T1D) in NOD mice and is currently in clinical trials in individuals with recent-onset disease. While research has demonstrated that imatinib protects islet $\beta$ cells from the harmful effects of ER stress, the role the immune system plays in its reversal of T1D has been less well understood, and specific cellular immune targets have not been identified. In this study, we demonstrate that B lymphocytes, an immune subset that normally drives diabetes pathology, are unexpectedly required for reversal of hyperglycemia in NOD mice treated with imatinib. In the presence of B lymphocytes, reversal was linked to an increase in serum insulin concentration, but not an increase in islet $\beta$ cell mass or proliferation. However, improved $\beta$ cell function was reflected by a partial recovery of expression of the transcription factor MafA, a sensitive marker of islet $\beta$ cell stress that is important for adult $\beta$ cell function. Imatinib treatment was found to increase the antioxidant capacity of B lymphocytes, improving ROS handling in NOD islets. This study reveals a mechanism through which imatinib enables B lymphocytes to orchestrate functional recovery of T1D $\beta$ cells.

Conflict of interest: The authors have declared that no conflict of interest.

Copyright: (c) 2019 American Society for Clinical Investigation

Submitted: October 3, 2018

Accepted: April 2, 2019

Published: April 9, 2019.

Reference information: /CI Insight. 2019;4(9):e125317. https://doi. org/10.1172/jici.insight.125317.

\section{Introduction}

T1D results from an absolute insufficiency of insulin, the hormone exclusively produced by islet $\beta$ cells to promote glucose uptake and storage in peripheral tissues. In the course of disease, adverse interactions between immune cells and islet $\beta$ cells lead to almost complete loss of $\beta$ cell mass in T1D. Successful strategies to ameliorate autoimmune destruction need to target both the immune system and islet cells, and identifying such strategies remains an important therapeutic goal. Imatinib (Gleevec) improves insulin responsiveness in the NOD animal model of T1D and is presently being evaluated in an ongoing phase II clinical trial in early-onset T1D patients (ClinicalTrials.gov, NCT01781975) (1). Although the mechanism of action is still unclear, imatinib appears to act by reducing stress levels in islet $\beta$ cells, which are proposed to be created in the islet under hyperglycemic and immune-infiltrating conditions (2-6). In the NOD mouse, the c-Abl tyrosine kinase promotes $\beta$ cell ER stress by enhancing IRE1 $\alpha$ activity, which drives the unfolded protein response, leading to ER stress and ultimately $\beta$ cell death. Imatinib is thought to impinge on this process by inhibiting c-Abl, thus preventing $\beta$ cell death (6). Although inhibiting c-Abl signaling through imatinib therapy leads to reduced $\beta$ cell stress, involvement of the immune system has not been identified as necessary for successful treatment.

While the preservation of islet $\beta$ cell function is a vital component of T1D treatment, it is unlikely to be sustained unless simultaneously targeting the underlying immune dysfunction. Prior studies have focused on the effects of imatinib on innate immune cells and $\mathrm{T}$ lymphocytes but it seems that these mechanisms are not at work in diabetes reversal (1, 7-12). B lymphocytes are also a critical immune target of imatinib in some clinical trials reported in oncology (13). This observation led us to hypothesize that B lymphocytes could also be immune targets of imatinib in diabetes treatment. This hypothesis is bolstered by the central antigen-presenting role that B lymphocytes play in T1D pathogenesis (14-21). In fact, the action of B lymphocytes is so pivotal to T1D development that autoantibodies produced by autoreactive B lymphocytes now define the earliest stage of the disease (termed stage 1), which precedes the occurrence of hyperglycemia (22). 
Our investigation demonstrates that imatinib improves NOD islet $\beta$ cell activity by elevating the antioxidant capacity of B lymphocytes that surround and invade the islets. The amelioration of oxidant stress allows the remaining islet $\beta$ cells to functionally recover, as revealed in part by improved expression of MafA, a redox-sensitive and functionally important $\beta$ cell-enriched transcription factor. This study defines an inducible mechanism of tissue protection by B lymphocytes, which may lead to novel therapeutic targets and opportunities to promote tissue recovery from autoimmune attack.

\section{Results}

Imatinib targets $B$ lymphocytes that are essential for diabetes reversal in NOD mice. To investigate the hypothesis that imatinib targets B lymphocytes in T1D, we analyzed splenocytes isolated from imatinib-treated and untreated prediabetic NOD mice. These mice were treated with imatinib mesylate $(1.5 \mathrm{mg} / \mathrm{mouse})$ or saline for 7 days via i.p. injection. A significant decrease in total splenocyte numbers was found in imatinib-treated mice, with B lymphocytes accounting for the majority of the lost cell numbers (Figure $1, \mathrm{~A}$ and $\mathrm{B}$ ). In contrast, no significant effect on $\mathrm{T}$ lymphocytes was observed, as previously reported (Figure 1B) (1). Additionally, imatinib treatment led to preferential apoptosis of B lymphocytes in ex vivo assays (Supplemental Figure 1; supplemental material available online with this article; https://doi. org/10.1172/jci.insight.125317DS1). Because imatinib only partially depleted the B lymphocyte population, we considered that its protective effect resulted from either depleting autoaggressive B lymphocytes or by enriching those producing a beneficial function among the remaining cells. We first assessed the capacity of imatinib to deplete insulin-reactive B lymphocytes in NOD.VH125 mice, which possess a small population of B cells that can bind insulin. We observed no significant decrease in insulin-reactive B lymphocytes (data not shown). Consequently, we used an animal model devoid of B lymphocytes to determine whether imatinib reverses diabetes by inducing a protective effect in B lymphocytes.

To examine the role of $\mathrm{B}$ lymphocytes in diabetes reversal, we utilized an immune cell transfer model of diabetes induction. In this model splenocytes were removed from a diabetic NOD WT donor mouse and depleted of all B lymphocytes via MACS-positive selection $\left(\mathrm{B} 220^{+}\right)$. The B lymphocyte negative fraction was then transferred into NOD.Rag1 $1^{-/-}$mice, which lack an adaptive immune system and develop diabetes only when donor NOD immune cells are transferred (Figure 1C). Blood glucose was monitored daily, and mice were assigned to one treatment group after 2 blood glucose readings of $>200 \mathrm{mg} / \mathrm{dl}$. Mice were then randomly assigned to imatinib therapy ( $1.5 \mathrm{mg} /$ mouse), imatinib therapy plus transfer of $20 \times 10^{6}$ splenocytes that were B lymphocyte depleted, or imatinib plus transfer of $20 \times 10^{6} \mathrm{~B}$ lymphocytes (purified by B220 $\mathrm{MACS}$ selection) from prediabetic NOD mice. As we found that normalization of blood glucose occurred very early in NOD WT mice reversed by imatinib (Supplemental Figure 2, A and B), we assessed diabetes reversal after 4 days on therapy. Diabetes was reversed only in mice that received B lymphocytes; none of the imatinib-treated mice without B lymphocytes demonstrated diabetes reversal (Figure 1D and Supplemental Figure 2C). As imatinib targets the c-Abl kinase, we next assessed the c-Abl signaling system in NOD B lymphocytes to determine whether these cells possessed a signaling profile that sensitized them to imatinib therapy.

$B$ lymphocytes in NOD mice have altered c-Abl signaling. Imatinib exhibits enhanced preference and inhibitory potency against the inactive form of c-Abl (23). The active conformation of c-Abl is induced by phosphorylation at tyrosine residue 412 (Y412). Since hyperphosphorylation of this residue imparts imatinib resistance in many BCR-ABL-driven cancers $(24,25)$, we hypothesized that reduced phosphorylation in $\mathrm{B}$ lymphocytes could explain increased sensitivity to apoptosis in response to imatinib in NOD mice. The B lymphocyte compartment is composed of multiple cell subsets, many of which contribute to T1D pathology and can respond differentially to imatinib therapy; for this reason we assessed c-Abl signaling in a cell subset-specific manner utilizing phosphoflow cytometry $(13,14,16,21,26-30)$. In B lymphocytes c-Abl is phosphorylated downstream of the B cell receptor (BCR) and CD19 signaling complex (31). Therefore, we assessed c-Abl phosphorylation (Y412) after stimulation of the BCR (anti-IgM Fab $)$ in both NOD and C57BL/6J (B6) mice.

Analysis of c-Abl phosphorylation revealed decreased c-Abl signaling in transitional B lymphocytes in NOD mice as compared with B6 mice (Figure 2, A and B, and Supplemental Figure 3A). Further analysis confirmed c-Abl pathway alterations in transitional B cells, as downstream proteins CrkL and PKC $\delta$ had reduced phosphorylation in comparison to marginal zone B lymphocytes (Supplemental Figure $3 \mathrm{~B}$ and data not shown). In contrast, c-Abl phosphorylation levels were similar in the marginal zone and marginal zone precursor cells. Follicular and immature B lymphocyte subsets demonstrated a lower capacity to 
A

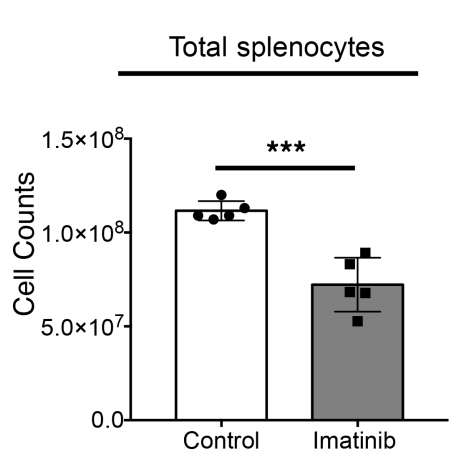

B

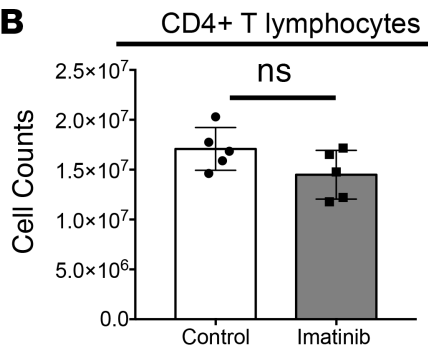

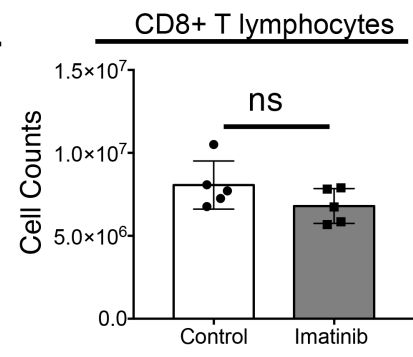

B lymphocytes

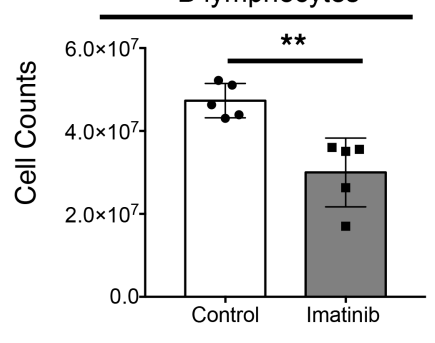

C
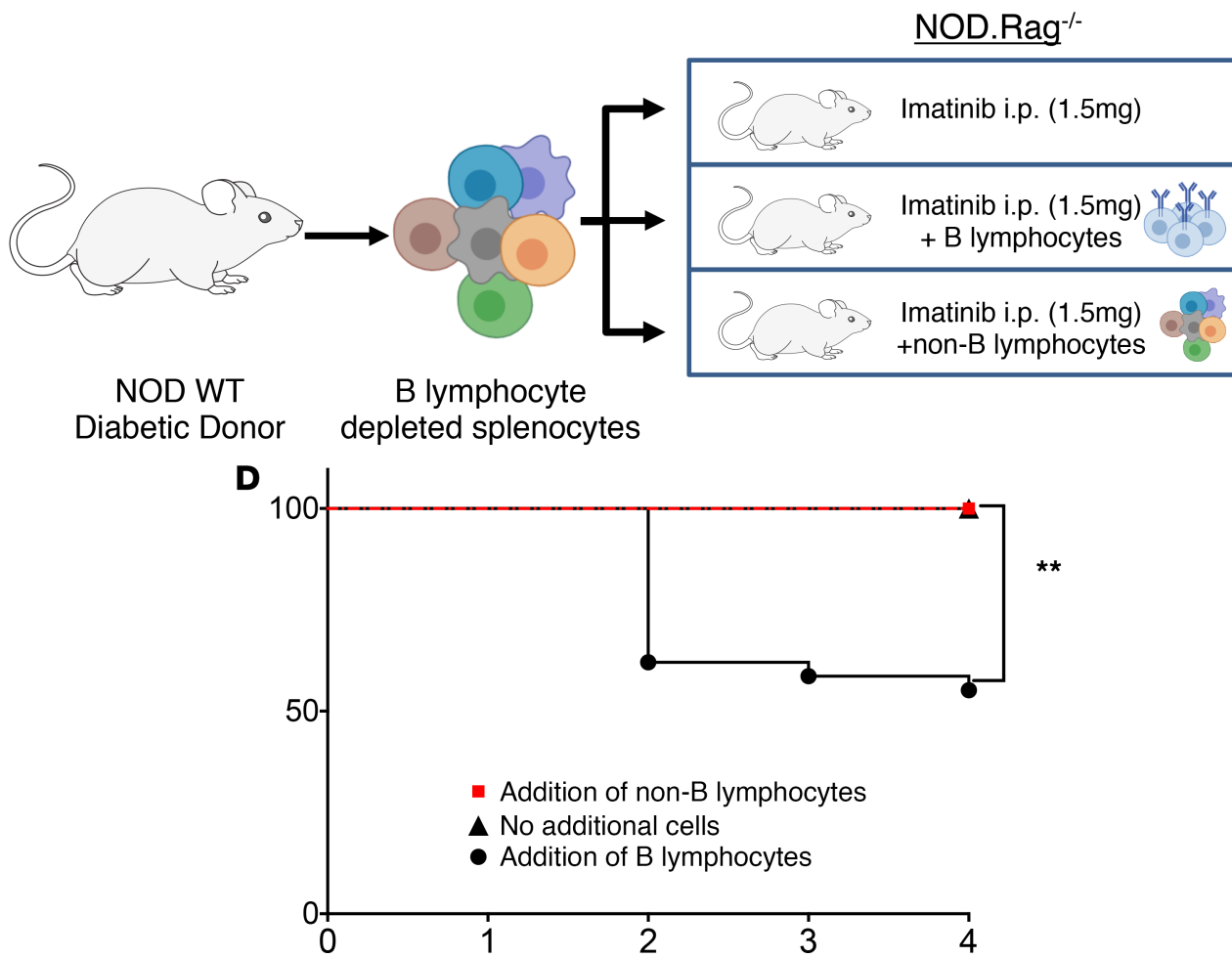

Figure 1. In vivo imatinib injections target B lymphocytes that are essential for diabetes reversal in NOD mice.

(A) Analysis of splenocytes revealed a reduction in total splenocyte numbers in NOD mice following imatinib treatment. ${ }^{* *} P=0.022$, Student's $t$ test. (B) B lymphocytes were preferentially depleted in NOD mice $\left(^{* *} P=0.041\right.$ Student's $t$ test) as compared with T lymphocytes ( $P=0.071$, Student's $t$ test). $n=5$ per group, representative of 5 experimental replicates. (C) B220 MACS-depleted splenocytes $\left(10 \times 10^{6}\right)$ from a diabetic NOD donor mouse were transferred into immunodeficient NOD.Rag1 $1^{-1-}$ mice. Mice were allowed to become diabetic, and at time of diabetes onset were given imatinib injections alone, $20 \times 10^{6} \mathrm{~B}$ cell-depleted splenocytes and imatinib, or $20 \times 10^{6}$ MACS-purified B lymphocytes and imatinib. (D) Blood glucose levels of diabetic mice on imatinib therapy revealed that only mice that received $B$ lymphocytes and imatinib together had normalization of blood glucose. $n=15$ in the group that received no additional cells, $n=6$ in the group that received additional non-B lymphocytes, $n=29$ in the group that received additional B lymphocytes. ${ }^{* *} P=0.002$, Mantel-Cox log-rank test. Data compiled from 5 separate experiments shown in $\mathbf{C}$. 
A

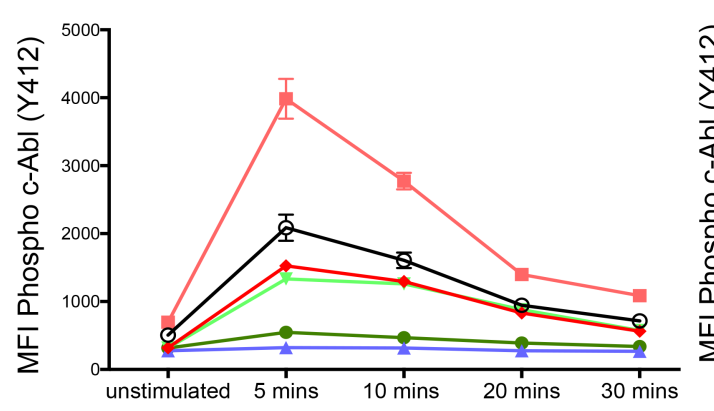

B

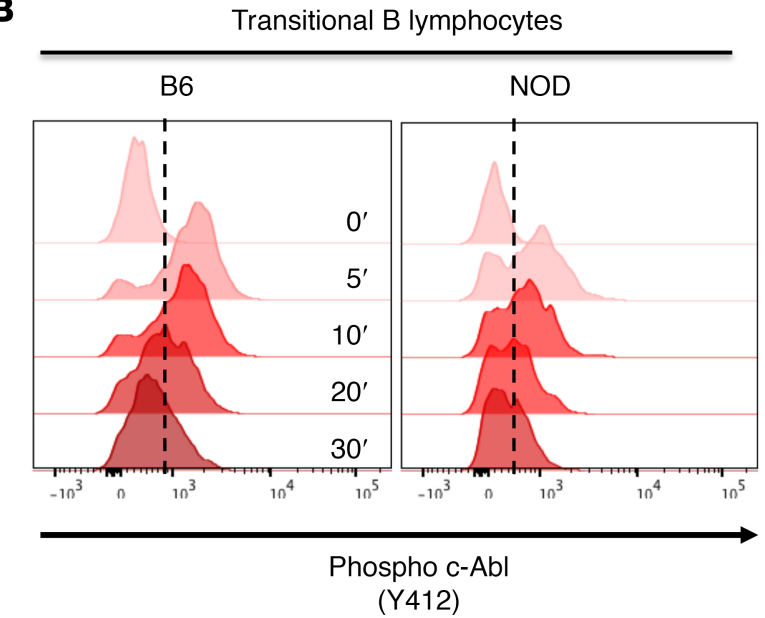

NOD B lymphocytes

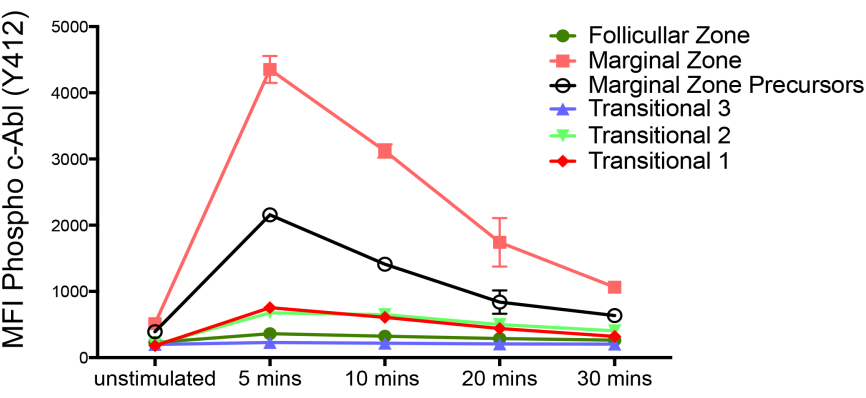

C

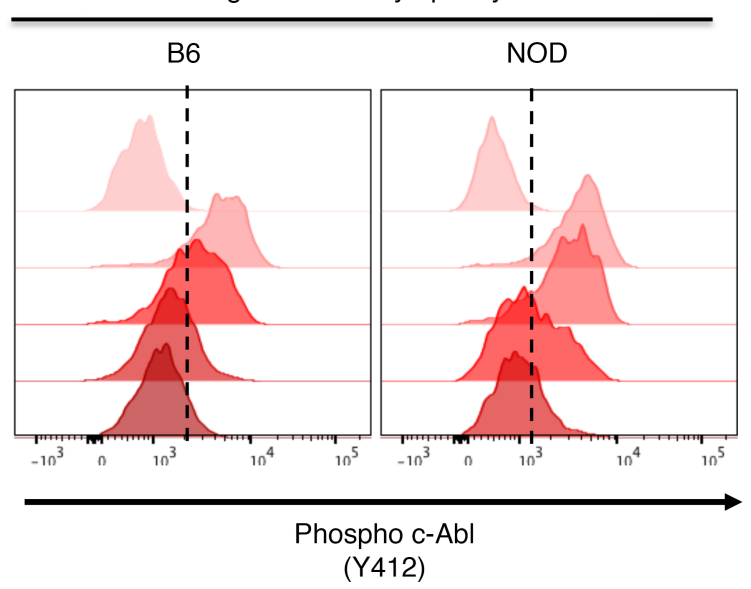

Figure 2. Specific NOD B lymphocyte subsets possess reduced c-Abl phosphorylation in response to BCR stimulation. (A) Splenocytes from B6 and NOD mice were stimulated with anti-IgM F(ab) $(10 \mu \mathrm{g} / \mathrm{ml})$ and subsequently fixed and stained for markers of B lymphocyte subsets and phosphorylated c-AbI(Y412) over a course of 30 minutes. Transitional and follicular B lymphocytes had reduced dynamic phosphorylation in both strains. c-Abl phosphorylation in transitional B lymphocytes from NOD mice was further reduced compared with B6 $(P<0.0001,1$-way ANOVA) (B), while marginal zone B lymphocytes were similar between strains (C). $n=3$ in each group, representative of 10 repeats. 0', 0 minutes.

signal through c-Abl in both strains, a potential indication that reduced c-Abl activity leaves these subsets sensitized to imatinib (Figure 2C and Supplemental Figure 3, A and B). These data suggest that imatinib may differentially affect c-Abl activity in B lymphocyte subsets.

Imatinib preferentially depletes $B$ lymphocyte subsets with reduced $c$-Abl signaling. Having identified that c-Abl signaling was lowest in follicular and transitional subsets, we hypothesized that these subsets would be preferentially depleted by imatinib therapy in NOD mice. We utilized flow cytometry to analyze the B lymphocyte compartment before and after imatinib therapy. Utilizing B220, IgM, CD19, CD21, and CD23, we were able to subset the splenic B lymphocyte compartment as described in a previous report (32). Analysis of mature B lymphocytes revealed preferential depletion of follicular B lymphocytes, while marginal zone B lymphocytes and their precursor population remained intact (Figure 3, A-C). Immature splenic B lymphocytes demonstrated preferential loss of transitional 1 and transitional 2 B lymphocytes, while transitional 3 B lymphocytes, largely thought to be an anergic population of B lymphocytes, were not impacted (Figure 3, D and E). In agreement with our signaling data, only those cells with the lowest c-Abl signaling activity were robustly depleted (compare Figure 2A with Figure 3). Nonetheless, depletion was incomplete, and we hypothesized that the remaining B lymphocytes may have resistance to imatinib therapy, a phenomenon characteristic of immune cells treated with imatinib in cancer studies (33-35). These alterations in B lymphocyte function could impact $\beta$ cell health in the islet, as B lymphocytes represent the most prominent component of the cellular islet infiltrate in NOD mice (36).

Imatinib restores $\beta$ cell function only in the presence of B lymphocytes. To understand how the islet cell population was impacted in imatinib-mediated, B lymphocyte-dependent reversal of diabetes, we analyzed 
A B lymphocytes

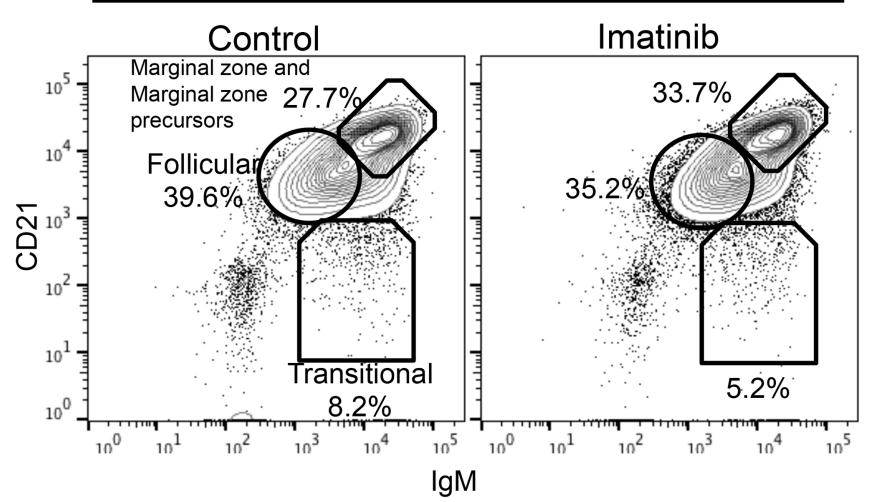

B

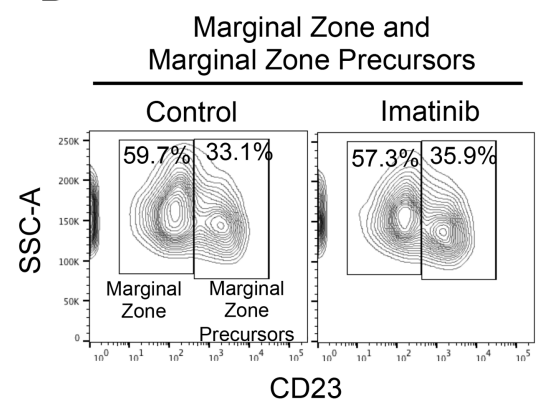

D

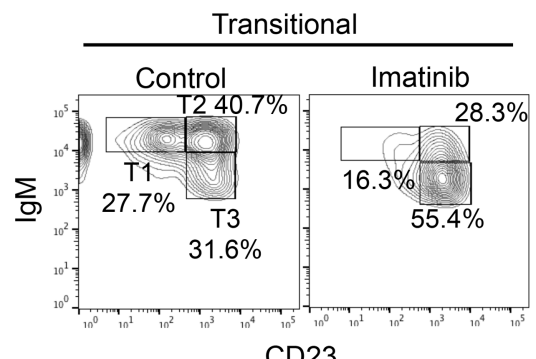

c

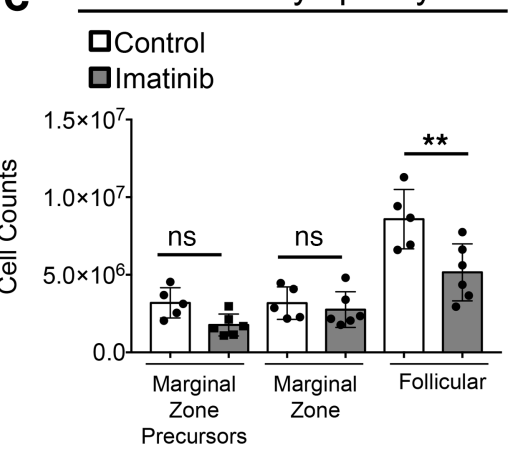

$\mathbf{E}$

Transitional B lymphocytes
Q Imatinib

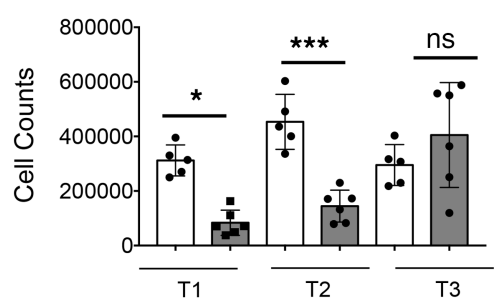

Figure 3. Imatinib preferentially depletes B lymphocyte subsets with reduced c-Abl signaling capacity. (A and C) Subset analysis of B lymphocytes revealed that among mature B lymphocytes, follicular B lymphocytes $(* * P=0.0031$, 2-way ANOVA followed by Šidák's multiple-comparisons test) were depleted. $n=6$ in each group. (B and C) Marginal zone (NS: $P=0.99,2$-way ANOVA followed by Šidák's multiple-comparisons test) and marginal zone precursors (NS: $P=0.51,2$-way ANOVA followed by Šidák's multiple-comparisons test) remained intact. $n=6$ in each group. (D and E) Immature B lymphocytes were also depleted, including preferential depletion of transitional 1 (T1) $\left({ }^{*} P=0.013,2\right.$-way ANOVA followed by Šidák's multiple-comparisons test) and T2 ${ }^{* *} P=0.005$, 2-way ANOVA followed by Šidák's multiple-comparisons test) subsets but not anergic T3 (NS: $P=0.51$, 2-way ANOVA followed by Šidák's multiple-comparisons test) B lymphocytes. ( $n=5$ controls and 6 imatinib treated). Data are representative of at least 5 repeats; group means are also shown on contour plots.

$\beta$ cells in imatinib-treated NOD.Rag1 $1^{-/}$mice in the presence or absence of B lymphocytes. We found that 4 days following imatinib treatment, neither the $\beta$ cell nor $\alpha$ cell area appeared changed (Figure 4 , A-C). Moreover, there was no obvious change in $\mathrm{Pax}^{+}$endocrine cells, proliferating insulin ${ }^{+} \mathrm{Ki}-67^{+}$cells, or apoptotic insulin ${ }^{+} \mathrm{TUNEL}^{+}$cells (Supplemental Figure 4A). Collectively, these results suggested that the rapid and robust improvement in glycemia in imatinib-treated mice with B lymphocytes reflected improved islet $\beta$ cell function, as further indicated by the relatively elevated serum insulin levels in fasted mice and relatively unchanged glucagon levels (Figure 4D and Supplemental Figure 4B).

Increased levels of ROS have been shown to deleteriously impact islet $\beta$ cell function, in part through reductions in $\beta$ cell-enriched transcription factors important in maintaining $\beta$ cell function. 
A

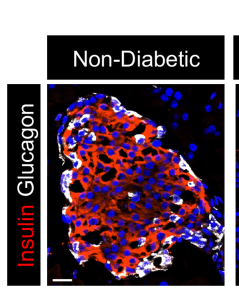

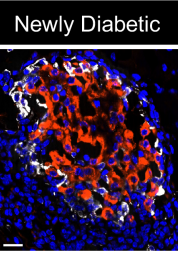

Imatinib treated
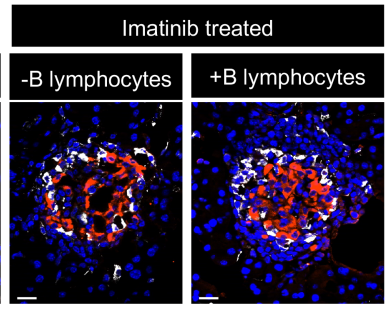

B

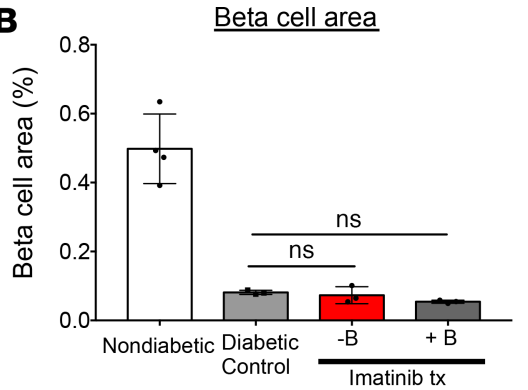

C

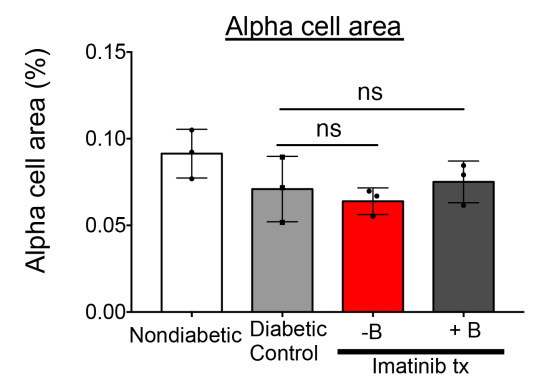

E
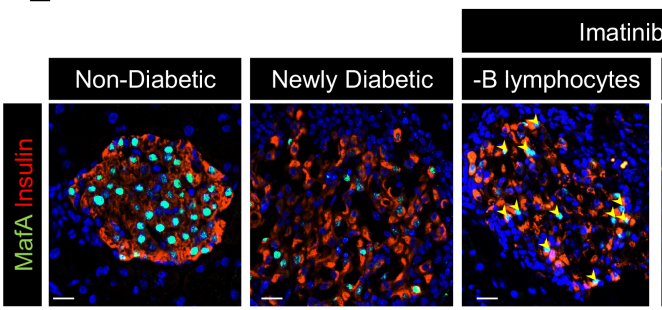

Imatinib Treated

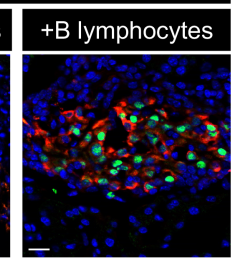

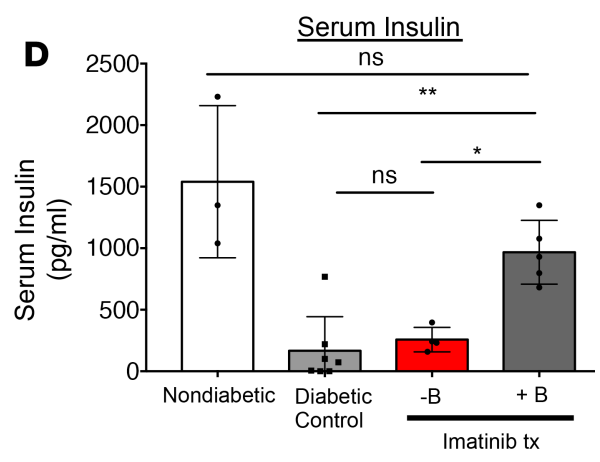

MafA+Beta Cell Area

$\mathbf{F}$

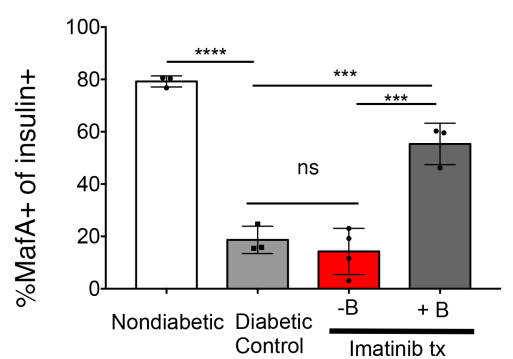

Figure 4. Imatinib therapy restores $\beta$ cell function but not $\beta$ cell mass in mice with B lymphocytes. (A) Pancreatic sections from WT, newly diabetic ( 2 consecutive blood glucose readings $>200 \mathrm{mg} / \mathrm{dl}$ ), and imatinib-treated (Imatinib tx) mice with or without B lymphocytes (4 days after treatment initiated) were evaluated for $\beta$ cell area and $\alpha$ cell area by staining with insulin- or glucagon-specific antibodies. (B and $\mathbf{C}$ ) Neither $\beta$ cell nor $\alpha$ cell area increased following imatinib treatment. $n=3$ in each group; 2-way ANOVA followed by Šidák's multiple-comparisons test. (D) Serum insulin levels were partially restored in NOD mice following imatinib treatment. ${ }^{* *} P=0.0032,{ }^{*} P=0.022$, 2-way ANOVA followed by Šidák's multiple-comparisons test. $n=3$ control, $n=7$ diabetic control, $n=5$ imatinib + B lymphocytes, and $n=4$ imatinib - B lymphocytes. (E) MafA and insulin staining of pancreatic sections reveals loss of MafA from insulin+ cells in newly diabetic mice. Only imatinib-treated mice with B lymphocytes restored MafA in insulin+ cells. (F) Quantification of MafA+insulin+ cells revealed partial recovery in imatinib-treated mice with B lymphocytes as compared to newly diabetic mice or imatinib-treated mice with no B lymphocytes. ( ${ }^{* * *} P<0.0001 ;{ }^{* *} P<0.0005,2$-way ANOVA followed by Šidák's multiple-comparisons test. $n=3$ in each group. Scale bars: $20 \mu \mathrm{m}$.

To understand the role B lymphocytes play in modulating $\beta$ cell function in imatinib therapy, we analyzed by immunofluorescence for markers of $\beta$ cell identity, function, and maturation in nondiabetic control mice, newly diabetic NOD mice, and NOD mice treated with imatinib with or without B lymphocytes (37-42). Levels of the Pdx1, Nkx6.1, and Nkx2.2 transcription factors (Supplemental Figure $5 \mathrm{~A}$ ) appeared unchanged in all conditions, whereas MafA was significantly reduced in newly diabetic and B lymphocyte-deficient NOD.Rag1 ${ }^{-/}$islet $\beta$ cells. Strikingly, MafA levels were partially recovered in insulin ${ }^{+}$cells of NODRag1 $1^{-/}$mice when imatinib therapy included B lymphocytes (Figure 4, E and F). MafA, a potent insulin-driving transcription factor important to adult $\beta$ cell function, is extremely sensitive to increased levels of oxidative stress (43). In contrast to MafA, urocortin 3 (Ucn3), a mature $\beta$ cell marker and hormone co-packaged within insulin secretory granules, did not recover following imatinib treatment (Supplemental Figure 5B) (37, 42, 44-46). The recovery of MafA and improvement in blood glucose homeostasis suggests that B lymphocytes act to relieve oxidant stress on $\beta$ cells, as observed in hematologic cells treated with imatinib but previously unrecognized in tissue recovery from autoimmune attack (33). 


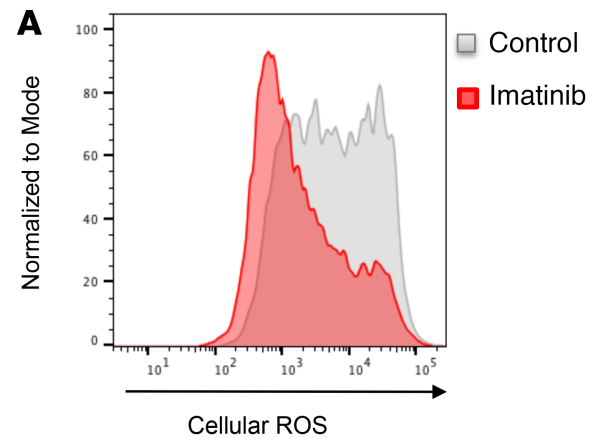

Cellular ROS

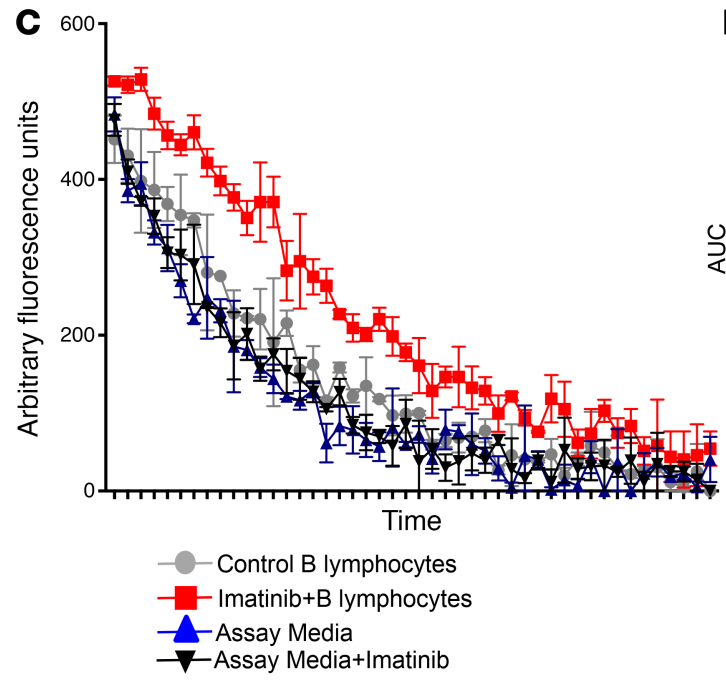

D

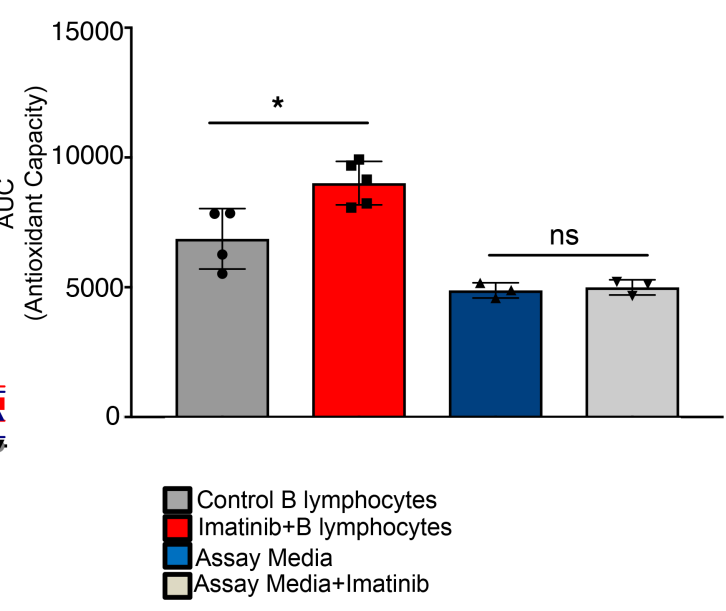

Figure 5. B lymphocytes acquire enhanced ROS handling capacity after imatinib therapy. (A and B) Cellular ROS analysis by flow cytometry of B lymphocytes from imatinib-treated and control NOD mice indicated that B lymphocytes from imatinib-treated mice possessed reduced cellular ROS (cROS). ${ }^{*} P=0.03$, Student's $t$ test. $n=5$. (C) B lymphocytes from NOD mice were purified by MACS and incubated in HBSS + FCS with or without imatinib (10 $\mu$ M). Supernatants were collected, and their antioxidant capacity was measured by a fluorescence quenching (hydroxyl radical antioxidant capacity [HORAC]) assay. Antioxidant capacity is revealed by prolongation of a ROS-sensitive fluorescent probe. Supernatants from imatinib-treated B lymphocytes prolonged fluorescence (red) as compared with B lymphocytes incubated with HBSS + FCS (gray), HBSS + FCS alone (blue), or HBSS + FCS + $10 \mu \mathrm{M}$ imatinib (black). (D) The area under the curve was calculated for biologic replicates and plotted, demonstrating a significant increase in secreted antioxidant capacity of $B$ lymphocytes ( ${ }^{*} P=0.015$, 2-way ANOVA followed by Šidák's multiple-comparisons test). Data are representative of at least 3 experimental repeats.

$B$ lymphocytes acquire enhanced ROS antioxidant capacity after imatinib therapy. Resistance to the effects of imatinib is a common problem encountered in the treatment of hematologic malignancies and is often associated with increased ROS handling proteins, including SODs and the glutathione system (33-35). We hypothesized that the remaining B lymphocytes enhance ROS handling to promote $\beta$ cell recovery of the ROS-sensitive transcription factor MafA following imatinib treatment. Although we observed no increase in the uptake of cystine, a precursor to glutathione assembly and a primary mechanism of imatinib-induced ROS regulation (Supplemental Figure 6A) (47), we did find a modest increase in SOD2 (i.e., the manganese-dependent superoxide dismutase) in both splenic and pancreatic B lymphocytes (Supplemental Figure 6, B-G), which reflects an activation of the B lymphocyte program to handle ROS at both sites. Interestingly, SOD2 expression was highest in the marginal zone at baseline, a potential second mechanism for their resistance to imatinib-induced depletion, whereas the transitional and follicular B lymphocytes demonstrated an increase in SOD2 after imatinib therapy (Supplemental Figure 6D). While this increase in SOD2 was modest and would not likely account for $\beta$ cell recovery by itself, we hypothesized that this change may be a sign of an improved overall ROS handling system in B lymphocytes, leading to a change in their antioxidant capacity.

To assess whether a change in overall ROS handling was induced by imatinib treatment, a cellular dye (H2DCFDA) that fluoresces with increasing levels of intracellular ROS was used. A robust decrease in intracellular ROS was found in the B lymphocytes of NOD mice treated with imatinib (Figure 5, A and B). Previous studies have demonstrated that lymphocytes can release antioxidant proteins to handle increased 
oxidative stress and that this is a mode by which B lymphocytes could provide antioxidant capacity to the surrounding $\beta$ cell microenvironment (48). Assaying cell supernatants from B lymphocytes incubated in media alone or in the presence of imatinib in the hydroxyl radical antioxidant capacity (HORAC) activity assay revealed that imatinib treatment increased ROS-neutralizing capacity in the media (Figure 5, $\mathrm{C}$ and D). These results explain how islet-invading B lymphocytes could extend tissue protection to nearby islet $\beta$ cells following imatinib treatment.

\section{Discussion}

Imatinib is an attractive therapeutic for T1D that can be delivered orally on an outpatient basis and has the potential to both promote $\beta$ cell recovery and establish longer-lasting $\beta$ cell protection from the immune system. Here we have uncovered an inducible interaction between the immune system and $\beta$ cells that allows the immune system to facilitate $\beta$ cell recovery. We have determined that imatinib partially depletes follicular mature and immature B lymphocyte populations and induces the remaining B lymphocytes to enhance their antioxidant capacity including secretion of antioxidants. $\beta$ Cells in the presence of these imatinib-treated B lymphocytes have partial restoration of MafA — to our knowledge a previously unobserved effect of imatinib within islet $\beta$ cells. Overall our data suggest a model in which local B lymphocytes under the influence of imatinib create a shift in oxidative stress that allows immediate $\beta$ cell recovery and leads to functional islet preservation.

Most studies in NOD mice have demonstrated that B lymphocytes are deleterious to $\beta$ cell health through priming of $\mathrm{T}$ lymphocytes to mediate $\beta$ cell death $(14-18,21)$. While the activity of B lymphocytes is central to the progression of $\mathrm{T} 1 \mathrm{D}$, this study illustrates a treatment that repurposes B lymphocytes to deliver islet-protective function. Anti-islet autoantibodies have long been recognized as the signature of new-onset disease. More recently, our understanding of these autoantibodies has evolved to reveal that they are present years to decades before the onset of dysglycemia and hence can be used to predict and even diagnose diabetes well in advance of clinical signs (22). These established clinical features are understood mechanistically in the NOD mouse model, where B lymphocytes are required for disease pathogenesis (16). In this model, islet-reactive B cells activate destructive $\mathrm{T}$ lymphocytes (14). These B lymphocytes are in fact required as antigen-presenting cells, and diabetes cannot proceed in their absence. Clinically, targeted B lymphocyte depletion has delayed loss of insulin C-peptide in patients with new-onset disease, but unfortunately disease progression returns once B lymphocytes repopulate (18-20, 49). Unlike current B lymphocyte-directed therapies, imatinib reprograms B lymphocytes with putative roles in pathology to instead orchestrate $\beta$ cell protection.

In our investigation, we found B lymphocytes that were most affected by imatinib possessed a reduction in the capacity to phosphorylate c-Abl at Y412, which favors a conformation amenable to imatinib binding. These cells were potently depleted, especially at the transitional zone stage, where autoreactive cells emerge to maturity and become capacitated to participate in the immune response. The cells that remained after imatinib treatment had enhanced redox handling capacity as revealed by their ability to neutralize oxidative challenge. Further evidence of change in their redox handling system was observed both in the form of expression of MnSOD (SOD2), which was seen in both spleen and pancreas, and in the ability to secrete antioxidants, thereby neutralizing local oxidants. Interestingly, some forms of MnSOD can be secreted, though we did not specifically assess that here, as there may be multiple factors contributing to the enhanced redox capacity (50). Further investigation is needed to determine what proteins and signaling pathways are necessary for this ROS response in NOD mice after imatinib treatment. The capacity for B lymphocytes to respond to imatinib by upregulating the redox system has been well established in the cancer literature, which describes use of imatinib for treatment of malignancies including B cell acute lymphoblastic leukemia (51). We did not observe any clear features of enhanced ROS production in B lymphocytes following imatinib, though this may have occurred prior to our measurements. The observed decrease in local ROS availability may also limit further T cell activation, as ROS can enhance $\mathrm{T}$ cell activation in an ongoing immune response $(52,53)$.

The $\beta$ cell is exquisitely sensitive to oxidative stress and has little of its own internal defense mechanisms to prevent oxidative injury (54-56). The MafA transcription factor is a very sensitive target of this oxidative stress, with levels being substantially reduced in T1D and T2D islets $(37,43,57)$. Here we observed a significant reduction in $\mathrm{MafA}^{+}$insulin ${ }^{+}$islet cells of hyperglycemic NOD mice, which was partially reversed in animals under imatinib therapy in the presence of B lymphocytes. This finding adds new insight into the previously established effects of imatinib at the level of the $\beta$ cell, which have focused more on the alleviation of ER stress and prevention of apoptosis $(1,6)$. Prior studies performed in vitro have established that imatinib 
can enhance insulin production through increasing levels of Nkx2.2 (5). We did not observe an increase in Nkx2.2 levels in vivo; however, we did similarly observe improved insulin levels following recovery. Imatinib has previously been shown to significantly reduced ER stress-mediated $\beta$ cell apoptosis in NOD islets prior to diabetes diagnosis (6). Here we did not observe a profound change in $\beta$ cell apoptosis, which could be due to the relatively late time point of assessment, after NOD mice were hyperglycemic. We predict, however, that the residual $\beta$ cells remaining, upon initiation of imatinib therapy, recover their functional status due to the enhanced ROS handling capacity of the pancreatic B lymphocytes, which positively affects MafA expression.

The translation of Gleevec (imatinib) to human clinical trials represents an exciting opportunity for utilization of an FDA approved drug that could be rapidly approved for use in humans with T1D. Our studies indicate a need to analyze the immune compartment with a focus on B lymphocytes in clinical studies and highlight what we believe to be a previously unobserved interaction between immune and $\beta$ cells. It is important to point out that our data indicate altered c-Abl signaling in B lymphocytes from NOD mice at baseline that could play a role in the predisposition of these cells to gaining ROS regulatory capacity. Our data suggest that NOD B lymphocytes are especially sensitive to imatinib therapy (Supplemental Figure 1), but it is unclear whether the same is true in humans with T1D. However, it is likely to be efficacious, since clinical data suggest that the ROS system is a universal target of imatinib in hematologic cells (33-35). As mounting evidence indicates T1D is a heterogenous disease, the cellular antioxidant response to imatinib may represent an important biomarker for predicting efficacy of imatinib therapy in patients with T1D.

\section{Methods}

Animals. C57BL6/J (B6), NOD/ShiLtJ (NOD), and immunodeficient NOD.Rag1 ${ }^{-/-}$mice were purchased from the Jackson Laboratory. Mice were housed in a specific pathogen-free facility at Vanderbilt University. B cell-deficient NOD. $\mu \mathrm{MT}$ mice were a gift from David Serreze (The Jackson Laboratory, Bar Harbor, Maine, USA).

Imatinib treatment and diabetes reversal. Imatinib mesylate (Eton Bioscience) was dissolved at a concentration of $1.5 \mathrm{mg} / 100 \mu \mathrm{l}$ saline. As the original studies in NOD mice utilized gavage to deliver Gleevec (imatinib) suspended in peanut oil, we first determined whether imatinib delivered i.p. was equally effective at reversing T1D (1). We determined that it was highly effective in reversing diabetes in newly diabetic NOD mice ( 2 consecutive blood glucose readings $>200 \mathrm{mg} / \mathrm{dl}$ ) (Supplemental Figure 2, A and B). In initial studies, $100 \mu \mathrm{l}$ imatinib was injected into diabetic NOD mice for up to 3 weeks. For studies involving cellular analysis, imatinib was injected for 5 days at a concentration of $1.5 \mathrm{mg} / 100 \mu 1$. NOD.Rag $1^{-/-}$mice were given $10 \times 10^{6}$ splenocytes depleted of B220 cells by MACS (Miltenyi Biotec, 130-049-501). Once diabetic, these mice were given $1.5 \mathrm{mg}$ imatinib in $100 \mu \mathrm{l}$ saline alone or concurrently with B lymphocytes $\left(20 \times 10^{6}\right)$, purified via $\mathrm{B} 220^{+}$selection.

Flow cytometry. Splenocytes or pancreatic lymphocytes were stained with the following fluorophore-conjugated antibodies: B220 (RA3-6B2), CD43 (S7), CD86 (GL1), CD40 (HM40-3), CD80, CD69 (H1.2F3), CD4 (RM4-5), CD8a (clone 53-6.7), CD25 (7D4), and IgM (II/41) purchased from BD Biosciences; and CD21 (7G6) and CD23 (B3B4) purchased from eBioscience. For intracellular staining, SOD1 (Abcam Polyclonal), SOD2 (Abcam Polyclonal), and c-Ab1 Y412 (247C7 Cell Signaling Technology) were utilized. In the case of intracellular staining, cells were fixed and permeabilized using the Foxp3/Transcription Factor Staining Buffer set (eBioscience, 00-5523-00) according to manufacturer's specifications. Cellular ROS was detected using H2DCFDA (Thermo Fisher Scientific) according to the manufacturer's recommendation. Cysteine conjugated to FITC was stained as previously described.

Phosphoflow measurement of c-Abl (Y412). Total splenocytes were isolated from NOD and B6 mice. These splenocytes were rested for 30 minutes at $37^{\circ} \mathrm{C}$ in DMEM culture media ( $10 \% \mathrm{FCS}$, penicillin/ streptomycin, and $2 \mathrm{ME}$ ). Following a rest, the cells were stimulated with an equal volume of media containing $10 \mu \mathrm{g} / \mathrm{ml}$ of anti-mouse IgM (Jackson ImmunoResearch Laboratories Inc.). At the end of the time course, cells were fixed with 1.5\% PFA at room temperature for 10 minutes, then washed and stained with extracellular subsets markers. Cells were then permeabilized with $0.1 \%$ Triton before being stained for phospho-c-Abl.

Tissue preparation and immunostaining. Pancreata were fixed in $4 \%$ (vol/vol) paraformaldehyde, embedded in O.C.T., and cut to $6 \mu \mathrm{m}$. Sections were blocked with $5 \%$ (vol/vol) normal donkey serum in $0.5 \%$ 
BSA/PBS (wt/vol) and incubated with primary antibodies overnight at $4^{\circ} \mathrm{C}$. Cyanine dye 2-(Cy2-), Cy3-, or Cy5-conjugated secondary antibodies (Jackson ImmunoResearch Laboratories Inc., 1:2000) were used for fluorescence detection. DAPI dye (SouthernBiotech) was used to detect nuclei in immunofluorescence images. The following primary antibodies were used: insulin (Dako, A056401-2; 1:1,000); glucagon (Cell Signaling Technology, 2760; 1:2000); Ki-67 mouse (BD Pharmingen, 550609; 1:1,000); MafA (Novus Biologicals, NBP1-00121; 1:500); Nkx6.1 (Novus Biologicals, NBP1-49672; 1:500); Ucn3 (Phoenix Pharmaceuticals, H-019-29; 1:1000); Pax6 (Covance, PRB-278P; 1:1000); Nkx2.2 (Santa Cruz Biotechnology Inc., sc-15015; 1:2000); and Pdx1 (gift from C. Wright, Vanderbilt University; 1:20,000). Images were collected on a Zeiss Axio Imager M2 fluorescence microscope.

For $\beta$ and $\alpha$ cell area measurements, 6 sections ( $\sim 240 \mu \mathrm{m}$ apart) were analyzed for insulin- or glucagon-positive area with HRP-secondary staining using the DAB substrate kit (Vector Laboratories) and counterstained with eosin. Images were collected on an Aperio ScanScope (Leica) whole slide scanner, and the percentage of $\beta$ and $\alpha$ cell area relative to whole tissue area (eosin) was calculated. TUNEL was performed on sections using the Click-iT TUNEL Assay kit (Thermo Fisher Scientific, C10617).

Measurement of serum insulin. Serum from fasted NOD.Rag1 $1^{-/}$mice was collected via retroorbital bleed. Serum was separated from platelets by centrifugation of whole blood. Insulin concentration was determined by Luminex assay by the Vanderbilt Hormone Assay and Analytical Services Core.

HORAC activity assay. Analysis of the antioxidant capacity of supernatants from imatinib-treated B lymphocytes was carried out according to the manufacturer's recommendation (Eagle Biosciences). Briefly, $10 \times 10^{6} \mathrm{~B}$ lymphocytes were incubated in $60 \mu \mathrm{HBSS}+1 \% \mathrm{FCS}$ for 5 hours at $37^{\circ} \mathrm{C}$ in the presence of 10 $\mu \mathrm{M}$ imatinib or with media only. As a control, wells with only $60 \mu 1 \mathrm{HBSS}+1 \%$ FCS with or without imatinib were incubated on the same plate for the same time period. At the end of the assay, cells were pelleted via centrifugation, and $20 \mu 1$ of supernatant was acquired for each well; all samples were assayed in duplicate. Supernatant was transferred to a black-walled optical plate, to which was added $120 \mu 1$ assay media, $20 \mu \mathrm{l}$ Fenton's reagent, and $20 \mu \mathrm{l}$ hydroxyl radical solution. Wells were thoroughly mixed, and fluorescence was measured every minute for 45 minutes on a fluorescence plate reader. As an assay control, gallic acid with a known antioxidant capacity was run on the same plate.

Statistics. Statistical analysis was performed with GraphPad Prism V5, using Student's $t$ test for comparison of 2 normally distributed conditions. One- or 2-way analysis of variance followed by Šidák's multiple-comparisons post hoc test was used to compare multiple groups. Statistical comparisons with $P \leq 0.05$ values were deemed significant. The error bars represent mean \pm SEM.

Study approval. All procedures were approved by the IACUC of Vanderbilt University prior to initiation of any experiments.

\section{Author contributions}

CSW, JMS, JK, BTS, EMH, RWS, and DJM designed, executed, and analyzed experiments. CSW, JMS, RWS, and DJM wrote the manuscript, which all authors reviewed.

\section{Acknowledgments}

This work was supported by NIH F31DK107321 (CSW), a JDRF Career Development Award (DJM), NIH R21AI121549 (DJM), and American Diabetes Association fellowship 1-16-PDF-109 (JMS). We would like to thank David Serreze for providing NOD. $\mu$ MT mice. We would like to thank Peter Siska for providing cysteine-conjugated to FITC. This work also utilized the cores of the Vanderbilt Diabetes Research and Training Center funded by grant DK020593 from the National Institute of Diabetes and Digestive and Kidney Disease.

Address correspondence to: Daniel J. Moore, Division of Endocrinology, Department of Pediatrics, Vanderbilt University Medical Center, 7415 MRB4, 2213 Garland Avenue, Nashville, Tennessee 37232, USA. Phone: 615.322.7427; Email: daniel.moore@vanderbilt.edu.

JMS's present address is: Department of Pediatrics, Indiana University School of Medicine, Indianapolis, Indiana, USA. 
1. Louvet C, et al. Tyrosine kinase inhibitors reverse type 1 diabetes in nonobese diabetic mice. Proc Natl Acad Sci USA 2008;105(48):18895-18900.

2. Hägerkvist R, Sandler S, Mokhtari D, Welsh N. Amelioration of diabetes by imatinib mesylate (Gleevec): role of beta-cell NF-kappaB activation and anti-apoptotic preconditioning. FASEB J. 2007;21(2):618-628.

3. Mokhtari D, Li T, Lu T, Welsh N. Effects of imatinib mesylate (Gleevec) on human islet NF-kappaB activation and chemokine production in vitro. PLoS ONE. 2011;6(9):e24831.

4. King AJ, Griffiths LA, Persaud SJ, Jones PM, Howell SL, Welsh N. Imatinib prevents beta cell death in vitro but does not improve islet transplantation outcome. Ups JMed Sci. 2016;121(2):140-145.

5. Xia CQ, et al. C-Abl inhibitor imatinib enhances insulin production by $\beta$ cells: $c-A b l$ negatively regulates insulin production via interfering with the expression of NKx2.2 and GLUT-2. PLoS ONE. 2014;9(5):e97694.

6. Morita S, et al. Targeting ABL-IRE1 $\alpha$ signaling spares ER-stressed pancreatic $\beta$ cells to reverse autoimmune diabetes [published erratum appears in Cell Metab. 2017;25(5):1207]. Cell Metab. 2017;25(4):883-897.e8.

7. Dietz AB, Souan L, Knutson GJ, Bulur PA, Litzow MR, Vuk-Pavlovic S. Imatinib mesylate inhibits T-cell proliferation in vitro and delayed-type hypersensitivity in vivo. Blood. 2004;104(4):1094-1099.

8. Appel S, et al. Effects of imatinib on monocyte-derived dendritic cells are mediated by inhibition of nuclear factor-kappaB and Akt signaling pathways. Clin Cancer Res. 2005;11(5):1928-1940.

9. Appel S, et al. Imatinib mesylate affects the development and function of dendritic cells generated from CD34+ peripheral blood progenitor cells. Blood. 2004;103(2):538-544.

10. Sinai P, Berg RE, Haynie JM, Egorin MJ, Ilaria RL, Forman J. Imatinib mesylate inhibits antigen-specific memory CD8 T cell responses in vivo. J Immunol. 2007;178(4):2028-2037.

11. Mumprecht S, Matter M, Pavelic V, Ochsenbein AF. Imatinib mesylate selectively impairs expansion of memory cytotoxic T cells without affecting the control of primary viral infections. Blood. 2006;108(10):3406-3413

12. Seggewiss R, et al. Imatinib inhibits T-cell receptor-mediated T-cell proliferation and activation in a dose-dependent manner Blood. 2005;105(6):2473-2479.

13. Carulli G, et al. Reduced circulating B-lymphocytes and altered B-cell compartments in patients suffering from chronic myeloid leukaemia undergoing therapy with Imatinib. Hematol Oncol. 2015;33(4):250-252.

14. Tian J, Zekzer D, Lu Y, Dang H, Kaufman DL. B cells are crucial for determinant spreading of T cell autoimmunity among beta cell antigens in diabetes-prone nonobese diabetic mice. J Immunol. 2006;176(4):2654-2661.

15. Henry RA, Kendall PL, Thomas JW. Autoantigen-specific B-cell depletion overcomes failed immune tolerance in type 1 diabetes. Diabetes. 2012;61(8):2037-2044.

16. Serreze DV, et al. B lymphocytes are essential for the initiation of T cell-mediated autoimmune diabetes: analysis of a new "speed congenic" stock of NOD.Ig mu null mice. J Exp Med. 1996;184(5):2049-2053.

17. Serreze DV, et al. Loss of intra-islet CD20 expression may complicate efficacy of B-cell-directed type 1 diabetes therapies. Diabetes. 2011;60(11):2914-2921.

18. Pescovitz MD, et al. Rituximab, B-lymphocyte depletion, and preservation of beta-cell function. N Engl J Med. 2009;361(22):2143-2152.

19. Yu L, et al. Rituximab selectively suppresses specific islet antibodies. Diabetes. 2011;60(10):2560-2565.

20. Pescovitz MD, et al. B-lymphocyte depletion with rituximab and $\beta$-cell function: two-year results. Diabetes Care. 2014;37(2):453-459.

21. Hulbert C, Riseili B, Rojas M, Thomas JW. B cell specificity contributes to the outcome of diabetes in nonobese diabetic mice. J Immunol. 2001;167(10):5535-5538.

22. Insel RA, et al. Staging presymptomatic type 1 diabetes: a scientific statement of JDRF, the Endocrine Society, and the American Diabetes Association. Diabetes Care. 2015;38(10):1964-1974.

23. Nagar B, et al. Crystal structures of the kinase domain of c-Abl in complex with the small molecule inhibitors PD173955 and imatinib (STI-571). Cancer Res. 2002;62(15):4236-4243.

24. Skaggs BJ, et al. Phosphorylation of the ATP-binding loop directs oncogenicity of drug-resistant BCR-ABL mutants. Proc Nat Acad Sci USA. 2006;103(51):19466-19471.

25. Nagar B. c-Abl tyrosine kinase and inhibition by the cancer drug imatinib (Gleevec/STI-571). J Nutr. 2007;137(6 Supp1 1):1518S-1523S

26. Mariño E, et al. Marginal-zone B-cells of nonobese diabetic mice expand with diabetes onset, invade the pancreatic lymph nodes, and present autoantigen to diabetogenic T-cells. Diabetes. 2008;57(2):395-404.

27. Kendall PL, Woodward EJ, Hulbert C, Thomas JW. Peritoneal B cells govern the outcome of diabetes in non-obese diabetic mice. Eur J Immunol. 2004;34(9):2387-2395.

28. Côrte-Real J, Duarte N, Tavares L, Penha-Gonçalves C. Innate stimulation of B1a cells enhances the autoreactive IgM repertoire in the NOD mouse: implications for type 1 diabetes. Diabetologia. 2012;55(6):1761-1772.

29. Zekavat G, et al. In vivo BLyS/BAFF neutralization ameliorates islet-directed autoimmunity in nonobese diabetic mice. J Immunol. 2008;181(11):8133-8144.

30. Catellani S, Pierri I, Gobbi M, Poggi A, Zocchi MR. Imatinib treatment induces CD5+ B lymphocytes and IgM natural antibodies with anti-leukemic reactivity in patients with chronic myelogenous leukemia. PLoS ONE. 2011;6(4):e18925.

31. Zipfel PA, Grove M, Blackburn K, Fujimoto M, Tedder TF, Pendergast AM. The c-Abl tyrosine kinase is regulated downstream of the B cell antigen receptor and interacts with CD19. J Immunol. 2000;165(12):6872-6879.

32. Quinn WJ, et al. Cutting edge: impaired transitional B cell production and selection in the nonobese diabetic mouse. J Immunol. 2006;176(12):7159-7164.

33. Reinke EN, Bera S, Diamond AM. Exposure of chronic myelogenous leukemia cells to imatinib results in the post-transcriptional induction of manganese superoxide dismutase. Leuk Lymphoma. 2015;56(4):1096-1099.

34. Milojkovic D, Apperley J. Mechanisms of resistance to imatinib and second-generation tyrosine inhibitors in chronic myeloid leukemia. Clin Cancer Res. 2009;15(24):7519-7527.

35. Tarumoto T, et al. Ascorbic acid restores sensitivity to imatinib via suppression of Nrf2-dependent gene expression in the imatinib-resistant cell line. Exp Hematol. 2004;32(4):375-381. 
36. Magnuson AM, Thurber GM, Kohler RH, Weissleder R, Mathis D, Benoist C. Population dynamics of islet-infiltrating cells in autoimmune diabetes. Proc Natl Acad Sci USA. 2015;112(5):1511-1516.

37. Hang Y, et al. The MafA transcription factor becomes essential to islet $\beta$-cells soon after birth. Diabetes. 2014;63(6):1994-2005

38. Gutiérrez GD, et al. Pancreatic $\beta$ cell identity requires continual repression of non- $\beta$ cell programs. J Clin Invest. 2017;127(1):244-259.

39. Schaffer AE, et al. Nkx6.1 controls a gene regulatory network required for establishing and maintaining pancreatic Beta cell identity. PLoS Genet. 2013;9(1):e1003274.

40. Mitchell RK, et al. The transcription factor Pax6 is required for pancreatic $\beta$ cell identity, glucose-regulated ATP synthesis, and $\mathrm{Ca}^{2+}$ dynamics in adult mice. J Biol Chem. 2017;292(21):8892-8906.

41. Gao T, et al. Pdx1 maintains $\beta$ cell identity and function by repressing an $\alpha$ cell program. Cell Metab. 2015;19(2):259-271.

42. van der Meulen T, Xie R, Kelly OG, Vale WW, Sander M, Huising MO. Urocortin 3 marks mature human primary and embryonic stem cell-derived pancreatic alpha and beta cells. PLoS ONE. 2012;7(12):e52181.

43. Guo S, et al. Inactivation of specific $\beta$ cell transcription factors in type 2 diabetes. J Clin Invest. 2013;123(8):3305-3316

44. van der Meulen T, et al. Urocortin3 mediates somatostatin-dependent negative feedback control of insulin secretion. Nat Med. 2015;21(7):769-776

45. van der Meulen T, Xie R, Kelly OG, Vale WW, Sander M, Huising MO. Urocortin 3 marks mature human primary and embryonic stem cell-derived pancreatic alpha and beta cells. PLoS ONE. 2012;7(12):e52181

46. Blum B, Hrvatin S, Schuetz C, Bonal C, Rezania A, Melton DA. Functional beta-cell maturation is marked by an increased glucose threshold and by expression of urocortin 3. Nat Biotechnol. 2012;30(3):261-264.

47. Siska PJ, et al. Fluorescence-based measurement of cystine uptake through $\mathrm{xCT}$ shows requirement for ROS detoxification in activated lymphocytes. J Immunol Methods. 2016;438:51-58.

48. Terrazzano G, et al. T cell activation induces CuZn superoxide dismutase (SOD)-1 intracellular re-localization, production and secretion. Biochim Biophys Acta. 2014;1843(2):265-274.

49. Chamberlain N, Massad C, Oe T, Cantaert T, Herold KC, Meffre E. Rituximab does not reset defective early B cell tolerance checkpoints. J Clin Invest. 2016;126(1):282-287.

50. Borrelli A, Schiattarella A, Bonelli P, Tuccillo FM, Buonaguro FM, Mancini A. The functional role of MnSOD as a biomarker of human diseases and therapeutic potential of a new isoform of a human recombinant MnSOD. Biomed Res Int. 2014;2014:476789.

51. Zelen I, et al. Antioxidant enzymes activities and plasma levels of oxidative stress markers in B-chronic lymphocytic leukemia patients. J BUON. 2010;15(2):330-336.

52. Franchina DG, Dostert C, Brenner D. Reactive oxygen species: involvement in T cell signaling and metabolism. Trends Immunol. 2018;39(6):489-502.

53. Thayer TC, et al. Superoxide production by macrophages and T cells is critical for the induction of autoreactivity and type 1 diabetes. Diabetes. 2011;60(8):2144-2151.

54. Padgett LE, Broniowska KA, Hansen PA, Corbett JA, Tse HM. The role of reactive oxygen species and proinflammatory cytokines in type 1 diabetes pathogenesis. Ann N Y Acad Sci. 2013;1281:16-35.

55. Tiedge M, Lortz S, Drinkgern J, Lenzen S. Relation between antioxidant enzyme gene expression and antioxidative defense status of insulin-producing cells. Diabetes. 1997;46(11):1733-1742.

56. Lenzen S, Drinkgern J, Tiedge M. Low antioxidant enzyme gene expression in pancreatic islets compared with various other mouse tissues. Free Radic Biol Med. 1996;20(3):463-466.

57. Harmon JS, Stein R, Robertson RP. Oxidative stress-mediated, post-translational loss of MafA protein as a contributing mechanism to loss of insulin gene expression in glucotoxic beta cells. J Biol Chem. 2005;280(12):11107-11113. 